\title{
MINERAL MAPPING USING CHANDRAYAAN-1 HYPERSPECTRAL (HYSI) DATA FROM MARE VAPORUM
}

\author{
Sayyad S. B. ${ }^{1}$, Mohammed Z. R. ${ }^{1 *}$, Deshmukh R. R. ${ }^{2}$ \\ ${ }^{1}$ Milliya Arts, Science \& management Science College, Beed, India - syedsb@ rediffmail.com \\ ${ }^{2}$ Dr. Babasaheb Ambedkar Marathwada University, Aurangabad, India - zeeeshan.shaikh@ gmail.com
}

Commission V, SS: Natural Resources Management

KEY WORDS: Imaging Spectroscopy, HySi,Compositional Mapping.

\begin{abstract}
:
The imaging spectroscopy offers an opportunity to map and discriminate different minerals on the lunar surface which further helps to understand the origin, evolution process, and the crustal composition on the surface of the moon. Compositional mapping of the lunar surface is considered as a standard approach for mineral mapping. This paper reports surface mineralogy of the lunar surface from Mare Vaporum using Chandrayaan-1 Hyperspectral remotely sensed data from HySi sensor. False color composite is created using different band shaping algorithms like band strength; band curve and band tilt parameters at crucial wavelength for spatial analysis. The Spectral analysis has been done by deriving reflectance spectra at varying locations from the area under study. The Study shows the mineral map with different categories of minerals which are high-Ca pyroxene and/or olivine and low Ca-pyroxene. However because of the limited spectral coverage of $\mathrm{HySi}$, data at the longer wavelengths required to discriminate among different group of minerals.
\end{abstract}

\section{INTRODUCTION}

Imaging spectroscopy considered to be a useful approach for understanding the evolution and composition of the earth and the planetary bodies. The hyperspectral data provides data in narrow contiguous bands with very fine spectral resolution. An approach which is based on color variations has been used as a standard and primary technique to study the composition of the earth and planetary bodies. Compositional mapping of the lunar surface can be done by deriving the false color composite image from the hyperspectral data. The moon minerals can be well identified and discriminated in visible to near infrared region of the electromagnetic spectrum by its characteristic absorption bands (Burns, 1970). The diagnostic absorption features for particular mineral are generally prominent in the near infrared region of the spectrum (Pieters, 1986). The specific characteristic absorption Features at various wavelengths across the electromagnetic spectrum is mainly because of electronic transitions and vibrational processes (Burns, 1993). Because of the compositional structure of the mineral, the shape and strength of the characteristic absorption and the wavelength position varies across the spectrum (Kramer, 2010, Anbazhagan, 2009 and 2010). Mineral mixing analysis of the lunar surface offers a platform to better understand the origin, crustal and overall evolution and geological history of the moon (Jin et al, 2013). The most abundant minerals on the lunar Surface are the iron bearing silicate minerals like olivine and pyroxene which has electronic transition absorption Band due to ferrous ion at near infrared wavelengths (Tompkins et.al,
1999). The Fig. 1 shows the library reflectance spectra of common mafic minerals found on the lunar surface. The spectra derived from RELAB database. The most common mafic minerals are pyroxene (Adams, 1974) and olivine has diagnostic absorption features in the near infrared region which varies with the composition of the mineral (Green, et al., 2011). The mineralogy of the lunar surface from different areas helps to predict the material composition. There were Many satellite missions which flown to moon with different objectives and applications. The Moon Mineralogy Mapper $\left(\mathrm{M}^{3}\right)$ (Tompkins et.al, 1999) and Hyperspectral Imager (HySi) (Kirankumar et al, 2009) onboard Chandrayaan-1, The India's first mission to moon (Goswami et al, 2009) provides hyperspectral data of the lunar surface. This paper demonstrates the compositional mapping of lunar surface to identify lunar surface minerology using Hysi data data which helps to understand the crustal composition of the lunar surface. A comparative study can also be done by considering data from both sensors which helps to discriminate among different minerals such as High-Ca pyroxene and olivine both have strong absorption near 1000 $\mathrm{nm}$, plagioclase has absorption at $1250 \mathrm{~nm}$ which is quite ambigous in case of $\mathrm{HySi}$ data due to its limited spectral coverage

\section{THE STUDY AREA}

The mare vaporum is located at the near side at southwest of mare serenitatis of the moon and centered at $13^{\circ} \mathrm{N}$ and $3^{\circ} \mathrm{E}$. The diameter is around $230 \mathrm{~km}$.

\footnotetext{
* Corresponding author
} 


\subsection{Data Set}

Hyperspectral Data from Hysi Sensor Onboard Chandrayaan-1 for mineral mapping from mare Vaporum was used in this study. The Hysi sensor captures the data in pushbroom mode covering 430-964 $\mathrm{nm}$ having 64 contigeous bands with the spectral resolution better than $15 \mathrm{~nm}$ and spatial resolution of 80 meters with $20 \mathrm{~km}$ swath (Kiran kumar et al, 2009). Band to band registered(BB-R) level-4 Hysi image was downloaded from issdc.gov.in. The image subset covering the study area from orbit number 1074 having product Id HYS_NREF_20090205T152648773 shown in Fig. 2. Environment for visualizing images (ENVI 5.3) software was used for processing, analyzing and deriving spectra from varying locations.

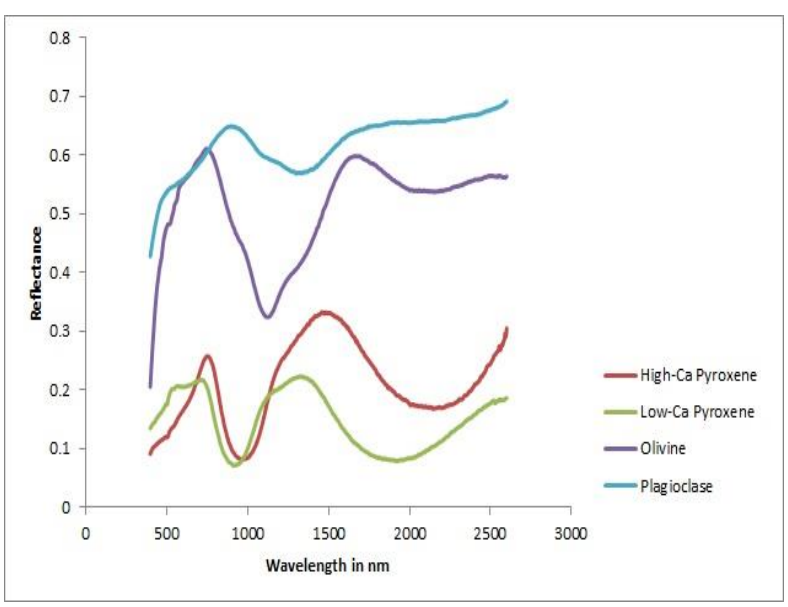

Figure 1. Reflectance Spectra of Typical Lunar Minerals.

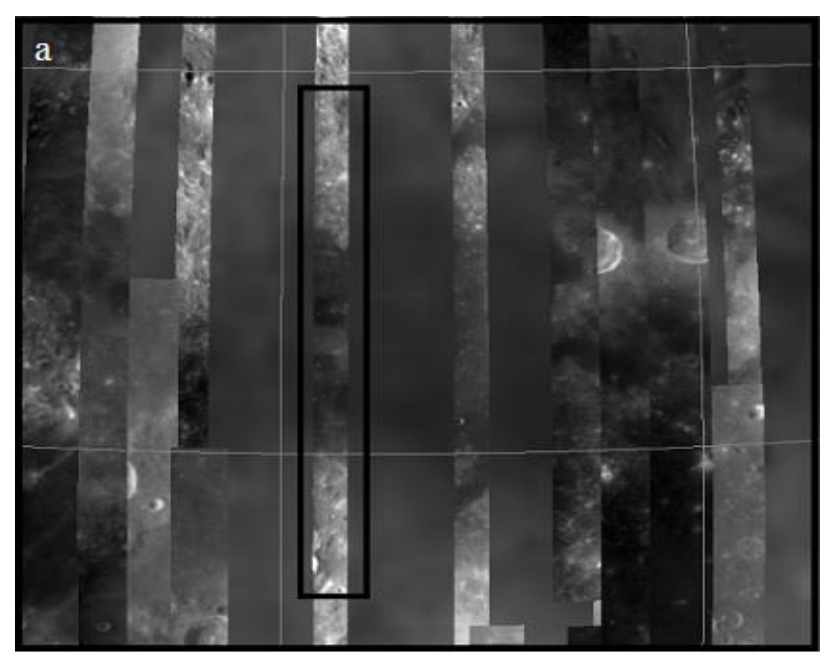

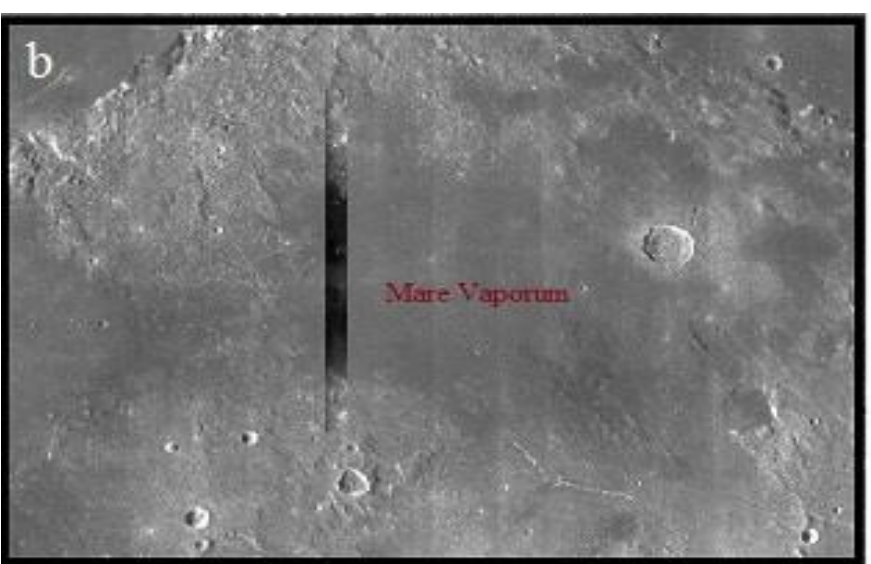

Figure 2. Hysi Coverage Shown on (a) globe view software from ISSDC and on (b) LRO LROC WAC Image.

\section{METHODOLOGY}

The band parameter used for mapping spatial variation more easily then the qualitative assessment. The false color composite derived from different band parameters for compositional mapping of lunar surface is a standard technique (Mccord and Adams 1973; Isaacson, Pieters, 2009; Pieters et al, 2001). Basically three band parameters which were originally used for UV-VIS data later modified and applied on the HySI data for lithological mapping of the lunar surface for mare Mascovience and interpreted different lithological units containing different mafic minerals (Bhattacharya et al, 2011). The different band parameters are band strength, band curve and band tilt which is used primarily for mapping the mafic minerals on the lunar surface. The subsequent section gives the detail about the band parameters.

\subsection{Band Strength}

The band strength parameter was primarily calculated by (Isaacson, Pieters, 2009) for clementine data to compositionally map the lunar surface is given by the equation (1) and later it was modified by (Bhattacharya et al, 2011). Because of the band limitation of hyperspectral imager sensor The band strength parameter helps to estimate the abundance of the mafic minerals by the analyzing the strength of the $1000 \mathrm{~nm}$ absorption band. The band strength is directly related to the depth of the absorption band and it is indicative of the relative presence of the mafic minerals and insensitive to the type of minerals.

$$
B S=R_{947.7} / R_{748.3}
$$

\subsection{Band Curve}

The band curvature is indicative of the type of the mafic mineral present and it is specifically sensitive to pyroxene composition. This parameter can be derived using HySI bands by the 
following equation suggested by (Bhattacharya et.al., 2011) that the curve decreases from low Ca pyroxene LCP bearing noritc rock to high $\mathrm{Ca}$ pyroxene $\mathrm{HCP}$ bearing gabbroic rock and it is further decreases for the olivine dominant rocks (Bhattacharya et al, 2011). The spectral Curve increases towards the longer wavelength as the wavelength of the absorption band moves towards the longer wavelengths.

$$
B C=\left(R_{748.3} / R_{906.3}\right)+\left(R_{947.7} / R_{906.3}\right)
$$

\subsection{Band Tilt}

The band tilt parameter is the intensity ratio and it is complementary to the band curvature as shown in the expression. The high values of band tilt show the area which is abundance of Clinopyroxene and olivine. The parameter defined by (Pieters et al, 2001) as difference in reflectance at crucial wavelengths and later modified by (Dhingra, , 2008) as the ratio of $900 \mathrm{~nm}$ and $1000 \mathrm{~nm}$. The formulation was also implemented on the HySI data by (Bhattacharya et al, 2011) and due to the band limitations of HySI it is further modified as in given in the expression.

$$
B T=R_{898.0} / R_{947.7}
$$

\section{DATA PROCESSING}

The Band to band registered level-4 data has been downloaded from orbit number 1074 for the study. The data product was opened in ENVI software by creating new header which was in radiance and The BBR-4 level HySI data then converted to apparent reflectance using the following equation.

$$
\frac{I}{F}=\frac{\pi L d^{2}}{F}
$$

Where $\mathrm{d}$ is sun moon distance, $\mathrm{F}$ is solar Flux measured in $\mathrm{W} / \mathrm{m}^{2} / \mu \mathrm{m}$ and $\mathrm{L}$ is radiance measured in $\mathrm{W} / \mathrm{m}^{2} / \mathrm{sr} / \mu \mathrm{m}$. The same process can be done in the ENVI software using the spectral math tool using the following expression by assigning variable s1 to HySI radiance image and s2 to available solar spectra. according to the yaw and limb direction information provided with the label file. The spatial subset of the HySI image is considered covering the western part of the area under study. Fig. 3 shows the subset of the HySI image. The areas in different colors are the reflectance spectra sampling locations. Around 50 reflectance spectra of $3 \times 3$ pixel averages were derived from spatially varying locations and only representative spectra were considered for further study. The average reflectance spectra from the spatially and spectrally varying areas are shown with different color markers which have been derived from the HySI data. Fig. 4 shows the reflectance spectra from the highlighted areas.

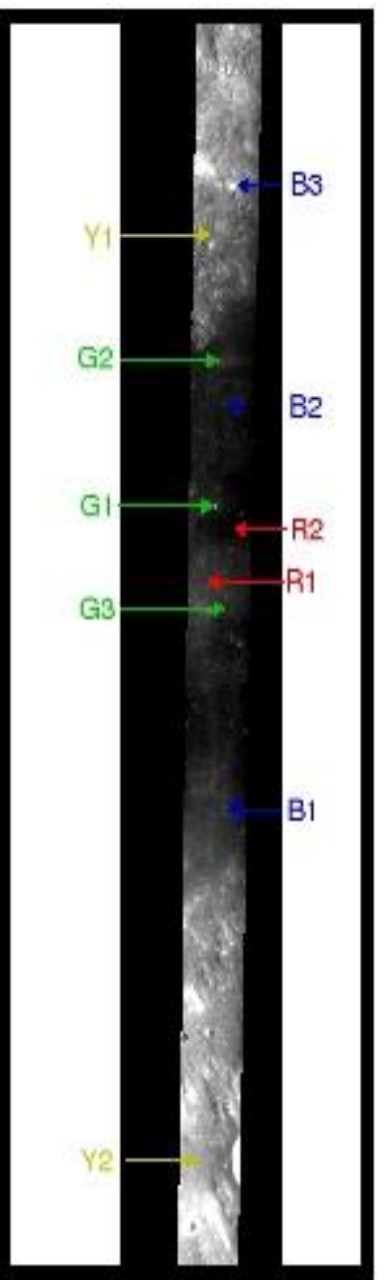

Figure 3. HySI Image Covering study area and representative locations from which reflectance spectra has been derived.

\section{$\frac{S_{1} * 1 d p i * 0.986161140705^{2}}{S_{z}}$}

(4)
The data set was geometrically corrected using geometry information provided in the geometry file with the data set. The orientation of the image changes after geometrical correction 

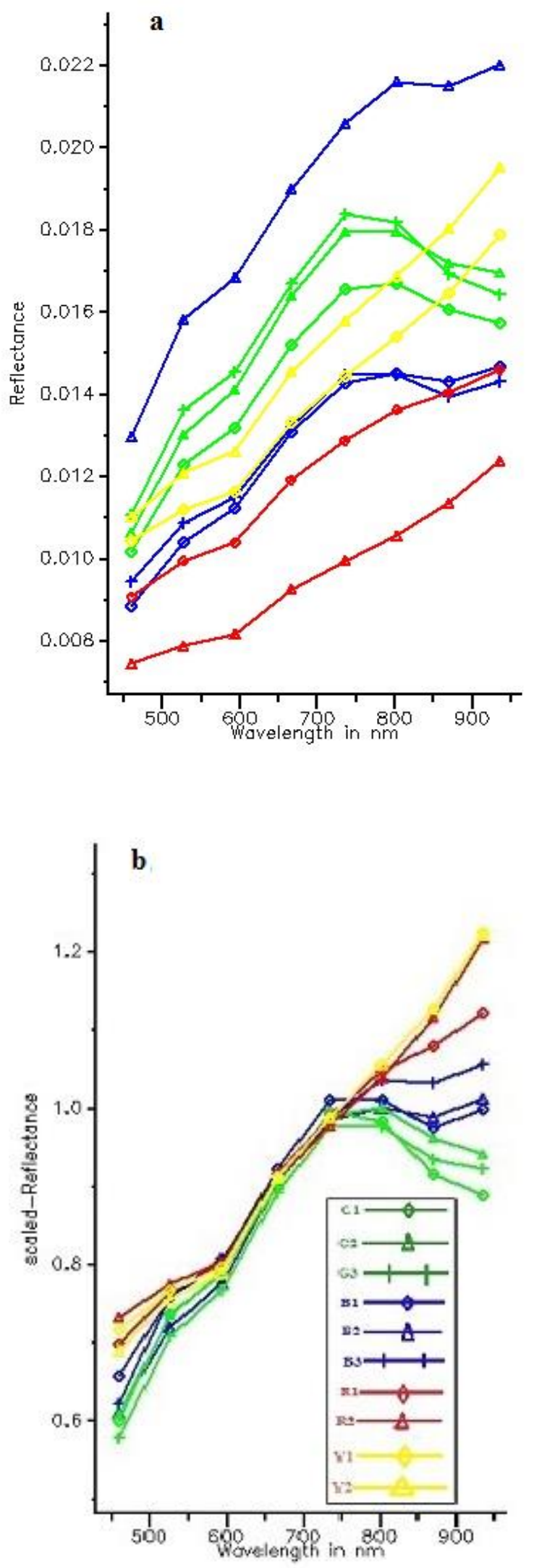

The different band parameters as discussed above like band strength, band curvature and band tilt was derived at particular wavelengths to identify the different mineral abundances like high-Ca pyroxene, low-Ca pyroxene, olivine and plagioclase within the study area. The color composite image was created by assigning band strength to blue color, band tilt to green color and band curve to red color in the color composite. Different minerals can be distinguished from the false color composite and as it groups the similar pixels it is also helpful in deriving the reflectance spectra

\section{RESULTS}

Fig (a) representing the raw reflectance spectra collected from different locations highlighted in Fig.3 and the Fig.4 (b) represents the normalized spectra at $748.3 \mathrm{~nm}$ for better representation. Fig. 5 shows the color composite image of the HySI data derived from different band shaping algorithm. high band strength values are appearing in shades of blue color indicating weathered rich rocks/anorthositic where blue color from highland (lower and upper part of the image) showing lack of $1000 \mathrm{~nm}$ absorption represented by Y1 and Y2 areas from highland basin soil in Fig(3) and the spectra for the same areas represented by spectra $\mathrm{Y} 1$ and $\mathrm{Y} 2$ can be seen in Fig (4) where steep slope can be observed probably because of the effect of space weathering which leads to reddened slope and reduction in overall reflectance. Similarly the steep slope can also be seen from mare area R1 and R2 as similar to the highland spectra Y1 and Y2. Space weathered area shows high band strength values (Tompkins et al, 1994; Borst et al, 2012) . High band curvature values indicate the presence of LCP rich areas which appears in the shades of red to pink. The areas B1,B2 and B3 from Fig(3) and the spectra derived from the area can be seen in Fig. (4). shows strong absorption feature near $900 \mathrm{~nm}$ indicative of LCP rich noritic rocks and can be observed at various locations of the image. The areas appearing in the shades of green to yellow representing the higher values of band tilt parameter. the areas G1,G2 and G3 and their reflectance spectra in Fig.4 shows strong absorption going towards the longer wavelengths which is beyond $964 \mathrm{~nm}$ indicating the probable presence of high-Ca pyroxene or plagioclase or olivine. However the limited spectral coverage of the HySi sensor, data at longer wavelength are required to confirm the presence and to discriminate the characteristic absorption but on the basis of the color composite and the reflectance spectra the different mafic rich areas can be predicted.

Figure 4. (a) raw Reflectance and (b) scaled reflectance spectra scaled at $748.3 \mathrm{~nm}$ derived from different locations. 


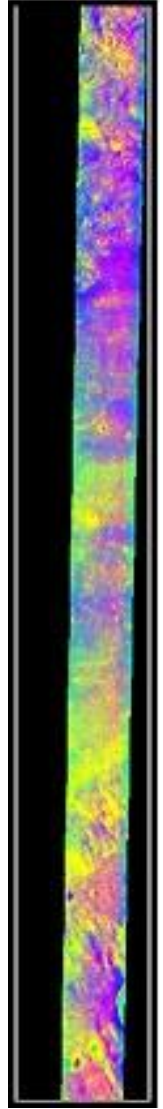

Figure 5. False Color Composite using BS, BT and BC

\section{CONCLUSION}

The mineral mixing analysis was carried out in various regions of mare Vaporum. It shows that creating the compositional map using different band parameters is the standard technique for mineral mapping. The hyperspectral data provides continuous reflectance spectra for spectral analysis which is useful for particular mineral identification. Areas that are rich in low-Ca (orthopyroxene) pyroxene mineral have been identified. Many Small fresh high-Ca (clinopyroxene) pyroxene craters are prominant in the mare region of and can be observed from the false color composite image. The absence of $900 \mathrm{~nm}$ absorption with steep slopes could be because the effect of high degree of space weathered soil. reflectance Spectra having strong absorption near $900 \mathrm{~nm}$ indicative of olivine and/or high Capyroxne and/or plagioclase, because of the limited spectral coverage of HySI, data at longer wavelengths are required for identification and discrimination of the mineralogy.

\section{ACKNOWLEDGEMENT}

The author is thankful for the financial assistance received from SAC-ISRO (India) under Chandrayaan-1 (AO). The research is based (partially or to a significant extent) on the results obtained from the Chandrayaan-1, first lunar mission of the Indian Space Research Organization (ISRO), archived at the Indian Space Science Data Center (ISSDC)"

\section{REFERENCES}

Adams J B (1974) visible and near infrared diffuse reflectance spectra of pyroxene as applied to remote sensing of solid objects in the solar system. $\mathrm{j}$ geophys. res $79,4829-4836$

Anbazhagan, S. and Arivazhagan, S. (2009) Reflectance spectra of analog basalts; implications for remote sensing of lunar geology. Planet. Space Sci., v.57, no.12, pp.13461358.

Anbazhagan, S. and Arivazhagan, S. (2010) Reflectance spectra of analog anorthosites: Implications for lunar highland mapping. Planet. Space Sci., v.58, no.5, pp.752760 .

Bhattacharya, S., Chauhan, P., Rajawat, A. S., Ajai and Kiran Kumar, A. S.,(2011). Lithological mapping of central part of Mare Moscoviense using Chandrayaan-1 Hyperspectral Imager (HySI) data

Borst, A.M., Foing, B.H., Davies, G.R., Van Western, W., (2012).Surface mineralogy and stratigraphy of the Lunar South Pole-Aitken basin determined from Clementine UV/VIS and NIR data. Planetary and Space Science 68 (1),76e85.

Burns, R. G., (1970) Mineralogical Applications of Crystal Field Theory, Cambridge University Press, New York

Burns, R. G. (1993). Mineralogical applications of crystal field theory (Vol. 5). Cambridge University Press

Dhingra, D., (2008). Exploring links between crater floor mineralogy and layered lunar crust. Adv. Space Res. 42 (2), 275-280. doi:10.1016/j.asr.2007.10.024

Green, R. O. et al., (2011).The Moon Mineralogy Mapper (M3) imaging spectrometer for lunar science: instrumentation, calibration, and on-orbit measurement performance. J. Geophys. Res., 116, E00G19

Goswami, J.N., Annadurai, M., (2009). Chandrayaan-1: India's first planetary science mission to the Moon. Lunar Planetary Science (CDROM) 40, 2571

Isaacson, P.J., Pieters, C.M., (2009). Northern Imbrium Noritic Anomaly. J. Geophys. Res. 114, E09007. doi:10.1029/2008JE003293

Jin, S., Arivazhagan, S. and Araki, H. (2013) New results and questions of lunar exploration from SELENE, Chang'E-1, Chandrayaan-1 and LRO/LCROSS. Adv. Space Res., v.52,no.2, pp.285-305. 
Kiran Kumar, A.S., Roy Chowdhury, A., Banerjee, A., Dave, A.B., Sharma, B.N., Shah, K.J., Murali, K.R., Mehta, S., Joshi, S.R., Sarkar, S.S., (2009). Hyper Spectral Imager for lunar mineral mapping in visible and near infrared band. Curr. Sci. 96 (4), 496-499.

Kramer, G.Y. (2010). Characterizing bedrock lithologies using small crater rim and ejecta probing (SCREP). Advances in Space Research. 45 (10), 1257-1267.

Mccord T.B and Adams J. B (1973) progress in remote optical analysis of lunar surface composition The Moon 7,453-474

Pieters, C. M. (1986), Composition of the lunar highland crust from near infrared spectroscopy, Rev. Geophys., 24(3), 557 - 578, doi:10.1029/RG024i003p00557.

Pieters, C.M., Gaddis, L., Jolliff, B., Duke, M., (2001). Rock types of South Pole-Aitken basin and extent of basaltic volcanism .J. Geophys. Res. 106,2800128022.doi:10.1029/2000JE001414.

Tompkins, S., Pieters, C.M., Mustard, J.F., Pinet, P.C., Chevrel, S.D., (1994). Distribution of materials excavated by the lunar crater Bullialdus and implications for the geologic history of the Nubium Region. Icarus 110, 261274.

Tompkins, S., Pieters, C.M., (1999). Mineralogy of the lunar crust: Results from Clementine. Meteor. Planet. Sci. $34,25-41$. 